\title{
Escala multidimensional aplicada aos estudos de apreciação musical ${ }^{1}$
}

\author{
Raquel Cocenas da Silva \\ José Lino Oliveira Bueno ${ }^{2}$ \\ Universidade de São Paulo, Ribeirão Preto-SP, Brasil \\ Emmanuel Bigand \\ Paul Molin \\ Université de Bourgogne, Dijon, França
}

\begin{abstract}
Resumo: A escala multidimensional (MDS) provém de uma família de técnicas de análise de proximidade de dados, obtida por meio do julgamento do participante que compara vários estímulos em vários traços, concomitantemente. A análise dos julgamentos de proximidade produz um espaço em que pontos representam a relação entre os estímulos em um espaço euclidiano. $\mathrm{O}$ ordenamento dos estímulos ao longo das dimensões neste espaço permite que inferências possam ser feitas sobre o universo perceptual subjacente ao grupo de pesquisa. A literatura mostra que a MDS vem ampliando o estudo da música e a mensuração de suas propriedades estruturais.
\end{abstract}

Palavras-chave: percepção, arte, música.

\section{Multidimensional scaling applied to studies of musical appreciation}

\begin{abstract}
The multidimensional scaling (MDS) originates from a set of techniques for analyzing proximity of data, which is obtained through the judgments of participants who concomitantly compare several stimuli in various dimensions. The analysis of proximity judgments produces an analysis in which points represent the relationship existent between stimuli in a Euclidean space. The configuration of stimuli in the space defined by these dimensions permits to make inferences about the underlying perceptual universe of the studied group. Literature reveals that MDS is enlarging the study of music and the measurement of its structural properties.
\end{abstract}

Keywords: perception, art, music.

\section{Escala multidimensional aplicada a los estudios de apreciación musical}

Resumen: La escala multidimensional (MDS) deriva de una familia de técnicas de análisis de proximidad de datos. Esta proximidad es obtenida a través del juicio de los participantes que comparan diversos estímulos con varias propiedades, al mismo tiempo. El análisis de la evaluación de proximidad produce un espacio en que los puntos representan la relación entre los estímulos en un espacio euclidiano. La clasificación de los estímulos a lo largo de las dimensiones en este espacio, permite realizar inferencias sobre el universo perceptivo del grupo de investigación. La literatura muestra que la MDS amplía el estudio de la música y la medición de sus propiedades estructurales.

Palabras clave: percepción, arte, música.

A adequação dos procedimentos estatísticos é uma das principais etapas para o desenvolvimento de uma pesquisa científica. É comum observarmos entre pesquisadores de diversas disciplinas algumas dificuldades para mensurar a estrutura de seu objeto de estudo. Isso ocorre principalmente quando a estrutura de um objeto encontra-se latente ou "escondida", quando mais de um fator subjacente é apropriado para a interpretação dos dados, ou mesmo quando a resposta dada pelos participantes pode não ser determinada por um único traço (Pasquali, 1999). Estas características geralmente estão presentes em um objeto com uma estrutura multidimensional, isto é, quando mais de uma dimensão subjacente é apropriada para levantar os dados. Desta maneira, correse o risco da interpretação não ser fidedigna por não alcançar todos os atributos embutidos nos dados, uma vez que as pré-concepções do experimentador podem estar refletidas na

1 Apoio: CAPES, CAPES-Cofecub, CNPq.

2 Endereço para correspondência:

Prof. Dr. José Lino Oliveira Bueno. Universidade de São Paulo. Faculdade de Filosofia, Ciências e Letras de Ribeirão Preto. Departamento de Psicologia e Educação. Av. Bandeirantes, 3900. CEP 14.040-901. Ribeirão Preto-SP, Brasil. E-mail: jldobuen@ffclrp.usp.br designação dos descritores verbais às várias dimensões, ou seja, as dimensões podem estar no universo conceitual do experimentador não no universo perceptual do observador (Silva \& Ribeiro-Filho, 2006).

Visando atingir uma acurácia na interpretação dos dados de objetos que apresentam multidimensionalidade de parâmetros, a técnica de escala multidimensional (MDS) provém de uma família de técnicas de análise de proximidade de dados e tem-se mostrado um importante instrumento matemático de mensuração. Esta técnica apresenta um caráter quantitativo e computacional e é desenvolvida por programas computacionais, Indscal, Alscal, Minissa, Multiscale, Mdscal, Clascal, Exscal (Schiffman, Reynolds, \& Young, 1981).

A escala multidimensional pode ser aplicada para diversos propósitos. Segundo Steyvers (2002), esta escala se aplica à análise exploratória de dados e para descobrir a representação mental do estímulo que explica como os julgamentos de similaridade são produzidos. A literatura mostra que ela vem sendo utilizada por psicólogos, sociólogos, antropólogos, economistas, educadores (Kruskal \& Wish, 1991), bem como na área de marketing, área social e ciências do comportamento (Chaturvedi \& Carroll, 2006). 
De acordo com Pasquali (1999), a técnica para levantar os dados de escalas multidimensionais consiste em solicitar ao participante que compare vários objetos (estímulos) em vários traços (parâmetros), concomitantemente, e não apenas avalie um objeto em um único traço de cada vez. A proximidade encontrada entre os objetos refletirá o grau de similaridade entre eles e será obtida a partir da distância entre os objetos de estudo (estímulos), os quais serão representados graficamente por meio de pontos em um espaço euclidiano ${ }^{1}$. Na prática, o espaço euclidiano é frequentemente usado devido à conveniência matemática nos procedimentos de MDS (Steyvers, 2002). A distância euclidiana entre um par de estímulos supondo um espaço bidimensional é representada por

$$
\mathrm{Dij}=\left[(\mathrm{Xi}-\mathrm{Xj})^{2}+(\mathrm{Yi}-\mathrm{Yj})^{2}\right]^{1 / 2}
$$

onde Xi e Xj são os valores de um ponto em uma coordenada e Yi e Yj são os valores de outro ponto em outra coordenada. Assim, as distâncias entre todos os pares de estímulos poderão ser calculadas (Silva \& Ribeiro-Filho, 2006).

Através das distâncias entre os pontos (pares de estímulos), os estímulos que forem julgados pelos participantes como similares serão representados por meio de pontos próximos, como em um mapa, ao passo que os estímulos julgados como não similares serão localizados com uma maior distância espacial.

Desta maneira, a partir da formação dos agrupamentos de estímulos julgados como similares, será possível identificar as dimensões, isto é, os eixos de coordenadas usados para localizar um ponto no espaço euclidiano. Uma dimensão permite visualizar características em comum partilhadas por uma porção significativa de dados. Essas dimensões serão determinadas pelo participante por meio de seu julgamento de similaridade entre os parâmetros multidimensionais dos estímulos. A MDS permite que os dados não sejam apenas representativos das percepções dos participantes ou das concepções do experimentador. Por não fornecer aos participantes um conjunto limitado de adjetivos ou escalas, este procedimento reduz a contaminação do pesquisador e é, portanto, uma metodologia relativamente sem constrangimento (Schiffman e cols., 1981).

A MDS não é uma forma de análise inferencial, ao contrário das estatísticas usuais como chi-square, t-tests, mas sim uma forma de análise indutiva em que o investigador busca descobrir o significado das dimensões que estrutura um domínio. Desta maneira, não existe uma solução apenas, mas o pesquisador pode escolher entre várias soluções que, em termos estatísticos, são iguais. Isso abre a oportunidade para uma mistura de métodos para interpretar melhor o significado dos resultados da MDS.

De acordo com Sturrock e Rocha (2000), os dados produzidos pela MDS são em geral representados por diagramas bidimensionais, permitindo uma melhor visualização, entretanto, muitos dados demandam três, quatro ou até mesmo cinco dimensões, sendo assim ajustados em duas dimensões. Para este tipo de visualização corre-se o risco da distância entre os pontos (pares de estímulos) estar ligeiramente incorreta e, para que a solução escolhida tenha uma boa correspondência com as similaridades entre os objetos avaliados originalmente, Kruskal (1964) propôs uma medida do grau de desvio entre as distâncias dij e as dissimilaridades observadas o $i j$ chamada de função do stress representada por

$$
S=\sqrt{\frac{\sum_{i j}\left(d_{i j}-d_{i j}^{*}\right)^{2}}{\sum_{i j} d_{i j}{ }^{2}}}
$$

Nesta equação, apenas a discrepância entre as distâncias preditas d $i j$ e as distâncias alvos d $i j^{*}$ é mensurada. De acordo com Steyvers (2002), as dissimilaridades observadas não aparecem, uma vez que "baseado na configuração real de pontos, as distâncias alvos dij são obtidas através de uma regressão monotônica e representam as distâncias monotonicamente relacionadas às dissimilaridades observadas" (p. 2).

Segundo Silva e Ribeiro-Filho (2006), "as discrepâncias entre estas distâncias ajustadas e as distâncias entre-pontos são então usadas para avaliar o grau de ajustamento ou bondade de ajuste da solução da MDS. Existem várias medidas de bondade de ajuste, das quais a mais comum é o stress" (p. 313). Uma informação adicional é identificar o limite superior e inferior do stress e comparar seu diagrama com este limite. Para um bom grau de ajustamento o valor de stress deveria resultar em zero, o que ocorreria para um estudo com uma pequena quantidade de objetos, porém, também é possível um bom grau de ajustamento com um valor de stress maior que zero, no caso de estudos com um maior número de objetos incluídos no estudo (Sturrock \& Rocha, 2000).

Este trabalho apresenta os conceitos centrais relacionados à técnica de escala multidimensional para que o leitor possa tomar conhecimento desta ferramenta e sua vasta aplicabilidade na pesquisa científica, ilustrado aqui através da resenha de alguns estudos que a utilizaram.

\section{Aplicações da escala multidimensional}

Na literatura especializada é possível ser encontrada uma diversidade de trabalhos abrangendo várias áreas de conhecimento. Esta técnica tem sido usada em pesquisa de Marketing (Chaturvedi \& Carroll, 2006), na Sociologia com estudos sobre posição social e disposição cultural (Han, 2003), no estudo da linguagem (Sardinha, 2000), na Psicofísica para mensuração da dor (Silva \& Ribeiro-Filho, 2006), na Psicologia Cognitiva (Shoben, 1983), entre outros.

Shimazuni (1998) realizou um estudo que contrastou a escrita de falantes nativos e não nativos. Os participantes foram divididos em dez brasileiros alunos de inglês como língua estrangeira (não-nativos), dez britânicos alunos de inglês (nativos) e dez jornalistas britânicos. Foram produzidos

${ }^{1}$ Espaço onde qualquer ponto pode ser definido em termos de um conjunto de coordenadas e a distância mais próxima entre dois pontos é uma reta. 
textos por estes participantes e, em seguida, os trinta textos foram submetidos à escala multidimensional. A análise sugeriu três dimensões, (1) expressão de organização textual ou densidade de informação; (2) expressão de posicionamento interpessoal e (3) relato de eventos. Os textos dos alunos nativos tiveram um escore médio maior na dimensão 1 , os textos dos alunos não-nativos tiveram maior escore na dimensão 2 e os textos dos jornalistas nativos na dimensão 3. Shimazuni (1998) concluiu que os resultados sugerem influência cultural e didática entre os alunos nativos e nãonativos, que demonstraram privilegiar aspectos interpessoais na escrita.

Shoben (1983) faz uma revisão na literatura das aplicações da MDS na Psicologia Cognitiva e cita Ross (1983) e o estudo realizado para classificar as teorias de memória da Psicologia Cognitiva em dimensões. A escala multidimensional foi aplicada a partir dos julgamentos de participantes especialistas nas doze conhecidas teorias de memória: o "distributed memory model" (Anderson, 1977), "HAM" (Anderson \& Bower, 1974), o "short-term memory model" (Atkinson \& Shiffrin, 1968), "levels of processing theory" (Craik \& Lockhart, 1972), o "propositional theory" (Kintsch, 1974), o "LNR model” (Norman \& Rumelhart, 1975), o “dual code theory" (Paivio, 1971), o "SAM model" (Raaijmakers \& Shiffrin, 1980), o "random walk model” (Ratcliff, 1978), o "schema model” (Rumellhart \& Ortony, 1977), o "MOPS model" (Schank, 1980) e "Tulving's (1975) memory model". Por meio do programa SINDSCAL, as teorias foram ordenadas em duas dimensões: (1) em termos de unidade de análise e (2) com relação ao grau de rigor da teoria. Na dimensão 1, o "MOPS model" e o "schema model" ficaram em um extremo da coordenada, e o "distributed memory model" e o "random walk model" ficaram em outro extremo. $\mathrm{Na}$ dimensão 2, as teorias com caráter matemático e computacional, como os modelos "LNR", "HAM", "SAM", "Ratcliff's model”, ficaram ordenadas em um extremo, e no outro as teorias não matemáticas, como "Tulving's model", "levels of processing" e a "dual code theory".

\section{Escala multidimensional no estudo da música}

De acordo com Grey (1977), a técnica de escala multidimensional mostra-se atrativa na investigação de estímulos extremamente complexos, como é o caso dos estímulos musicais, por não exigir do pesquisador a necessidade de levantar uma hipótese a priori para a construção física de seus estímulos. Isto é possível, pois com os julgamentos perceptuais de similaridade emitidos pelos participantes sobre uma diversidade de estímulos naturais, há a possibilidade de explorar os vários fatores que contribuíram para a relação da distância subjetiva. Estes fatores podem ser parâmetros físicos dos estímulos, que então conduziriam a um modelo psicofísico; contudo, a técnica da escala multidimensional pode igualmente descobrir outros fatores envolvidos em estratégias do julgamento.
Em cognição musical, a MDS tem sido usada para investigar a estrutura de respostas emocionais para peças musicais (Bigand, Vieillard, Madurell, Marozeau, \& Dacquet, 2005), representação mental do timbre musical (Caclin, Mc Adams, Smith, \& Winsberg, 2005; Faure; Mc Adams, 1997; Grey, 1977), fundamentos cognitivos da frequência musical (Krumhans1, 1990), escala multidimensional de estilos de música e estilo de exemplares de piano (Eastlund, 1992; Thorisson, 1998), escala multidimensional e música ocidental (Hevner, 1935; Juslin, 2001; Pollard-Gott, 1983), música e cérebro (Zatorre, 2003), entre outros.

Caclin e cols. (2005) investigaram a relevância perceptual de parâmetros acústicos do timbre através de julgamentos de dissimilaridade. Segundo estes autores, a literatura provê vários correlatos acústicos de timbre-espaço dimensionais. Trinta participantes ouviram dezesseis estímulos musicais de som sintético para se familiarizarem com o campo de variação musical. Após uma prova de dez escolhas randomizadas foi calculada a taxa de dissimilaridade para todos os possíveis pares dos dezesseis sons. As taxas de dissimilaridade foram feitas clicando com o mouse em uma escala apresentada na tela do computador com pontos nomeados "igual" e "diferente". Os julgamentos de dissimilaridade foram analisados através de dois programas de escala multidimensional (CLASCAL E CONSCAL). Os parâmetros "attack time", "spectral centroid" e "spectrum fine structure" apareceram como os mais determinantes do timbre, delimitando assim uma dimensão. Já o "spectral flux” apareceu como o parâmetro menos saliente, delimitando outra dimensão.

Outro exemplo da utilização da MDS é o estudo de Krumhansl (1990) sobre hierarquias tonais. Na música ocidental a altura, os acordes e a tonalidade pertencem a uma complexa relação que se refere à hierarquia tonal/harmônica ocidental. Entre 1979 e 1990, Krumhansl e colaboradores realizaram uma série de pesquisas para investigar como as hierarquias tonais/harmônicas ocidentais eram representadas mentalmente (Krumhansl, 1990). A complexidade da estrutura da altura ocidental é devida ao fato de que os eventos musicais (tons e acordes) têm funções musicais diferentes dependendo da tonalidade de contexto em que ocorrem. Esta função depende das distâncias psicológicas existentes entre as 24 tonalidades maiores e menores. O método de análise multidimensional permite representar esta grande série de relações abstratas através do espaço toroidal ${ }^{1}$, em que são expressas as hierarquias entre tons e acordes, as distâncias psicológicas entre as 24 tonalidades musicais, bem como, a maneira como as hierarquias entre tons e acordes mudam de acordo com a tonalidade de contexto.

Eastlund (1992), em sua pesquisa sobre escala multidimensional e estilos musicais, comparou as dimensões perceptuais dominantes emergentes através dos julgamentos de dissimilaridade de 30 ouvintes de música divididos entre músicos e não músicos durante a classificação de trechos musicais. Neste estudo, foram utilizados trechos musicais de 15 segundos cada, representativos de música tonal européia, 
compostos entre o período de 1762 e 1896. Os dados de cada subgrupo (músicos e não-músicos) foram analisados através de escala multidimensional resultando em uma solução tridimensional. As coordenadas de ambos os subgrupos foram significativamente correlacionadas permitindo a fusão dos dados. As dimensões encontradas foram (1) período histórico; (2) complexidade ou quantidade de informação e (3) tempo.

Pollard-Gott (1983) trabalhou com passagens de peças clássicas e escala multidimensional, e, em seu experimento, solicitou aos participantes a escuta de uma sonata de Liszt por três vezes, uma em cada sessão. A cada sessão os participantes eram incentivados a pensarem e a tomarem nota sobre as passagens. Após cada sessão os participantes julgaram a similaridade de 28 pares de estímulos que foram produzidos por oito passagens musicais. Os dados de dissimilaridade foram analisados pelo programa SINDSCAL e a cada sessão surgiram dimensões diferentes. Após a primeira, as dimensões emergentes remeteram a fatores de percepção de escuta mais simples, como feliz-triste, alto-baixo, simplescomplexo e forte-suave. Após a segunda sessão, a dimensão identificada foi uma dimensão temática. Observou-se que à medida que os participantes avançavam nas sessões e obtinham mais conhecimento sobre as passagens musicais, mais emergia a dimensão temática, possivelmente decorrente de uma percepção um pouco mais refinada em detrimento da observada na primeira sessão de escuta.

O estudo de Bigand e cols. (2005) ilustra a utilização de escalas multidimensionais identificando dimensões entre 27 trechos de música erudita representativos da música ocidental. A escolha dos estímulos foi embasada em trechos provenientes de obras com instrumentação solo, música de câmara e orquestra, representativas dos diferentes períodos da música ocidental, como o barroco, clássico, romântico e moderno. Os participantes escutaram os trechos musicais, focaram a atenção na emoção induzida e agruparam os trechos que haviam induzido emoções similares. Após ser analisado o número de grupos formados por cada participante, os dados foram submetidos à escala multidimensional, programa EXSCAL MDS, a fim de serem verificadas as possíveis dimensões psicológicas emergentes. Os autores encontraram duas dimensões psicológicas principais que influenciam as respostas emocionais para música erudita ocidental: arousal (estado de excitação fisiológica) e valência afetiva. A Figura 1 apresenta a solução geométrica para os 27 trechos musicais resultantes da MDS com duas das dimensões obtidas (arousal e valência afetiva).

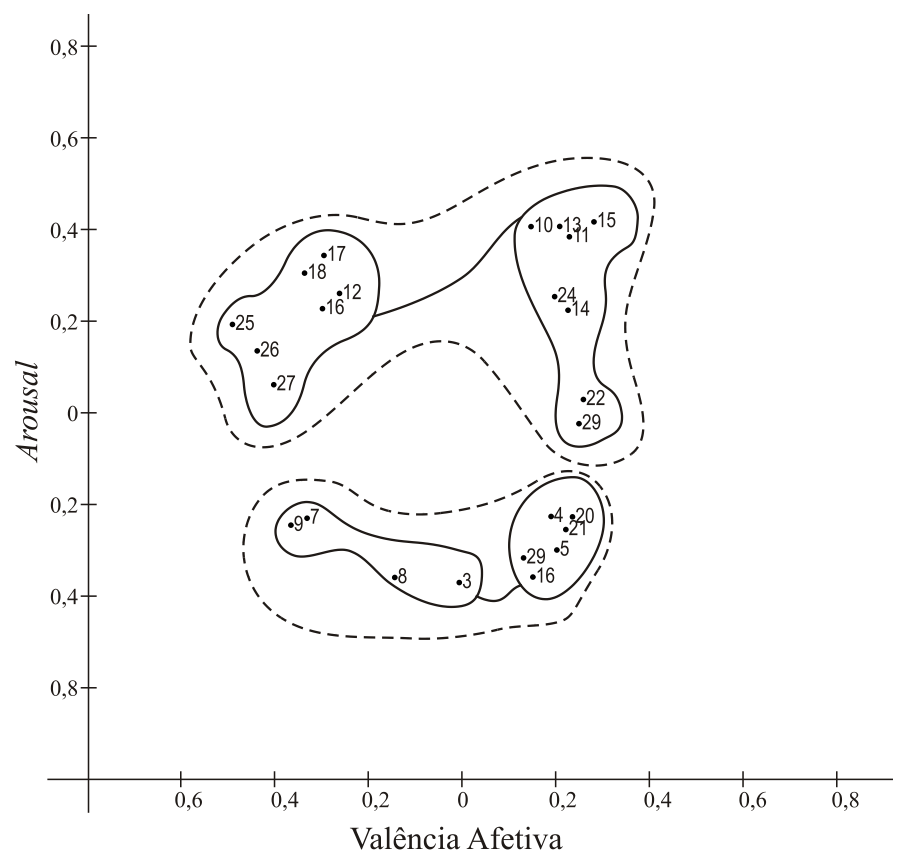

Figura 1. Adaptada de Bigand e cols. (2005), apresenta a solução geométrica para 27 trechos musicais resultantes da MDS com duas dimensões obtidas (arousal e valência afetiva identificadas através do eixo vertical e horizontal). Apresenta também uma análise de clusters (primário, linhas tracejadas; secundário, linha contínua).

\section{Considerações finais}

A arte possui um conteúdo formatado, que concentra a junção de aspectos racionais e emocionais, objetivos e subjetivos (Bilbão \& Cury, 2006). A música compõe o universo das obras de arte e o estudo experimental de composições musicais permite o acesso a estes conteúdos de forma singular e suas influências sobre aspectos cognitivos, como por exemplo, o estudo da percepção subjetiva de tempo.

Não há na literatura estudos utilizando a escala multidimensional relacionando estímulos musicais com a percepção subjetiva de tempo. Investigações vêm mostrando que distintos parâmetros das composições musicais podem ser utilizados como marcadores temporais pelos indivíduos. 
A manipulação experimental desses marcadores, como as escalas musicais (Bueno \& Ramos, 2007), as modulações de trechos musicais distantes e próximos (Firmino \& Bueno, 2008), o contexto rítmico (Jones \& Boltz, 1989) e as variações tonais nos finais musicais (Lelis, 2002) mostraram que distintos processos participam da percepção temporal.

Face à diversidade de parâmetros empregados nos estudos de tempo subjetivo e apreciação musical, a utilização de uma escala multidimensional como método de pesquisa em estudos da percepção temporal poderia trazer novas informações sobre o tempo subjetivo. Ao delimitar as dimensões a partir dos múltiplos parâmetros ou marcadores temporais presentes na estrutura de composições musicais, é possível que se identifique um ou mais parâmetros responsáveis pela alteração na percepção subjetiva de tempo (Cocenas-Silva \& Bueno, 2008).

A contribuição para a pesquisa científica, com a introdução de mais um método de mensuração do tempo subjetivo, é o conhecimento mais amplo e sistematizado das dimensões dos estímulos acústicos, do tipo musicais genuínos, associados à percepção subjetiva de tempo.

\section{Referências}

Anderson, J. A. (1977). Neural models with cognitive applications. In D. LaBerge \& S. J. Samuels (Eds.), Basic processes in reading: Perception and comprehension. Hillsdale, NJ: Erbaum.

Anderson, J. R., \& Bower, G. H. (1974). Human associative memory. Washington, DC: Wiston.

Atkinson, R. C., \& Shiffrin, R. M. (1968). Human memory: A proposed system and its control processes. In K. W. Spence \& J. T. Spence (Eds.), The psychology of learning and motivation: Advances in research and theory, 2. New York: Academic Press.

Bigand, E., Vieillard, S., Madurell, F., Marozeau, J., \& Dacquet, A. (2005). Multidimensional scaling of emotional responses to music: The effect of musical expertise and of the duration of the excerpts. Cognition and Emotion, 19, 1113-1139.

Bilbão, G. G. L., \& Cury, V. E. (2006). O artista e sua arte: Um estudo fenomenológico. Paidéia (Ribeirão Preto), 16, 91-100.

Bueno, J. L., O. \& Ramos, D. (2007). Musical mode and estimation of time. Perceptual and Motor Skills, 105, 1087-1092.

Caclin, A., McAdams, S., Smith, B. K., \& Winsberg, S. (2005). Acoustic correlates of timbre space dimensions: A confirmatory study using synthetic tones. Journal of the Acoustical Society of America, 118, 471-482.

Chaturvedi, A., \& Carroll, J. D. (2006). CLUSCALE (CLUstering and multidimensional Scal[E] ing): A Three-Way Hybrid Model Incorporating Overlapping Clustering and Multidimensional Scaling Structure. Journal of Classification, 23, 269-299.
Cocenas-Silva, R., \& Bueno, J. L. O. (2008, abril). Análise multidimensional dos parâmetros de estímulos musicais subjacentes à estimação temporal de peças clássicas. Trabalho apresentado no III Congresso Interno de Psicobiologia, Ribeirão Preto, SP.

Craik, F. I. M., \& Lockhart, R. S. (1972). Levels of processing: A framework for memory research. Journal of Verbal Learning and Verbal Behavior, 11, 671-684.

Eastlund, J. O. (1992). A multidimensional scaling analysis of musical style. Journal of Research in Music Education, 40, 204-215.

Faure, A., \& Mc Adams, S. (1997, abril). Comparaison de profils sémantiques et de l'espace perceptif de timbres musicaux. Trabalho apresentado no Congrès Français d'Acoustique, Marseille, França.

Firmino, E. A., \& Bueno, J. L. O. (2008). Tonal modulation and subjective time. Journal of New Music Research, 37, 275-297.

Grey, J. M. (1977). Multidimensional perceptual scaling of musical timbres. Journal of the Acoustical Society of America, 61, 1270-1277.

Han, S.-K. (2003). Unraveling the brow: What and how of choice in musical preference. Sociological Perspectives, 46, 435-459.

Hevner, K. (1935). The affective character of the major and minor modes in music. American Journal of Psychology, 47, 103-118.

Jones, M. R., \& Boltz, M. G. (1989). Dynamic attending and responses to time. Psychological Review, 96, 459-491.

Juslin, P. N. (2001). Communication emotion and music performance: A review and theoretical framework. In P. N. Juslin \& J. A. Sloboda (Eds.), Music and emotion theory and research (pp. 309-337). New York: Oxford University Press.

Kintsch, W. (1974). The representation of meaning in memory. Hillsdale, NJ: Erlbaum.

Krumahnsl, C. (1990). Cognition foundation of musical pitch. Oxford, UK: Oxford University Press.

Kruskal, J. B. (1964). Multidimensional scaling by optimizing goodness of fit to a nonmetric hypothesis. Psychometrika, 29, 1-27.

Kruskal, J. B., \& Wish, M. (1991). Multidimensional scaling. Newbury Park, CA: Sage Publications.

Lelis, C. M. C. (2002). Influência de audições musicais com variação de composição sobre estimação subjetiva de tempo. Dissertação de mestrado não-publicada, Universidade de São Paulo, Ribeirão Preto, SP.

Norman, D. A., Rumelhart, D. E., \& The LNR Research Group (1975). Explorations in cognition. San Francisco, CA: Freeman.

Paivio, A. (1971). Imagery and verbal processes. New York: Holt, Rinehart \& Winston.

Pasquali, L. (Org.). (1999). Instrumentos psicológicos: Manual prático de elaboração. Brasília, DF: LabPAM. 
Pollard-Gott, L. (1983). Emergence of thematic concepts in repeated listening to music. Cognitive Psychology, 15, 66-94.

Raaijmakers, J. G. W., \& Shiffrin, R. M. (1980). SAM: A theory of probabilistic search of associative memory. In G. H. Bower (Ed.), The psychology of learning and motivation, 14. New York: Academic Press.

Ratcliff, R. (1978). A theory of memory retrieval. Psychological Review, 85, 59-108.

Rumelhart, D. E., \& Ortony, A. (1977). The representation of knowledge in memory. In R. C. Anderson, R. J. Spiro, \& W. E. Montague (Eds.), Schooling and the acquisition of knowledge. Hillsdale, NJ: Erlbaum.

Sardinha, T. B. (2000). Análise multidimensional. DELTA, 16, 99-127.

Schank, R. C. (1980). Language and memory. Cognitive Science, 4, 243-284.

Schiffman S. S., Reynolds, M. L., \& Young, F. W. (1981). Introduction to Multidimensional Scaling: Theory, methods and applications. New York: Academic Press.

Shimazumi, M. (1998, julho). Investigating EFL writing: A multidimensional analysis. Trabalho apresentado na VI Convenção Braz-TESOL, Recife, PE.

Silva, J. A., \& Ribeiro-Filho, N. P. (2006). Avaliação e mensuração de dor: Pesquisa, teoria e prática. Ribeirão Preto, SP: FUNPEC.

Shoben, E. J. (1983). Applications of multidimensional scaling in cognitive psychology. Applied Psychological Measurement, 7, 473-490.

Sturrock, K., \& Rocha, J. (2000). A Multidimensional scaling stress evaluation table. Field Methods, 12, 49-60.

Steyvers, M. (2002). Multidimensional scaling. In Encyclopedia of cognitive science (pp. 1-5). Stanford, CA: Stanford University.

Thorisson, T. (1998). Comparison of novice listener's similarity judgments and style categorization of classic and romantic piano examplars. Psychology of Music, 26, 186-196.

Tulving, E. (1975). Ecphoric processes in recall and recognition. In J. Brown (Ed.), Recall and recognition. London: Wiley.

Zatorre, R. J. (2003). Music and brain. Annals New York Academy of Sciences, 999, 4-14.

Raquel Cocenas da Silva é Mestre em Ciências pelo Programa de Pós-graduação em Psicobiologia da Faculdade de Filosofia, Ciências e Letras de Ribeirão Preto da Universidade de São Paulo.

José Lino Oliveira Bueno é Professor Titular da Faculdade de Filosofia, Ciências e Letras de Ribeirão Preto da Universidade de São Paulo, bolsista de produtividade em pesquisa do CNPq.

Emmanuel Bigand é professor da Universidade de Bourgogne, Dijon, França.

Paul Molin é professor da Universidade de Bourgogne, Dijon, França. 